\title{
Spontaneous emission of an atom in front of a mirror
}

\author{
Almut Beige*, Jiannis Pachos, and Herbert Walther \\ Max-Planck-Institut für Quantenoptik, D-85748 Garching, Germany
}

(October 29, 2018)

\begin{abstract}
Motivated by a recent experiment [J. Eschner et al., Nature 413, 495 (2001)], we now present a theoretical study on the fluorescence of an atom in front of a mirror. On the assumption that the presence of the distant mirror and a lens imposes boundary conditions on the electric field in a plane close to the atom, we derive the intensities of the emitted light as a function of an effective atom-mirror distance. The results obtained are in good agreement with the experimental findings.
\end{abstract}

PACS: 42.50.Lc, 03.65.Yz, 42.50.Ct

\section{INTRODUCTION}

One of the fundamental subjects in quantum optics is describing the fluorescence from single atom sources. Different scenarios have been considered, the simplest one referring to an atom in free space [1]. The fluorescence of an atom can be altered for example by the presence of other atoms inducing dipole-dipole interactions [2], by the presence of a mirror [3-6] or by the single mode of the electromagnetic field inside a cavity $[7,8]$. To investigate experimentally these phenomena ion trapping technology has been employed and good agreement with theoretical predictions has been found.

Theoretical models have been developed starting from the Hamiltonian that describes the atom, the free radiation field and their interaction. To predict the time evolution of an ensemble of atoms, master equations can be derived by tracing over all possible photon states. Alternatively, it can be assumed that the environment performs continuous measurements on the free radiation field. This leads to a quantum trajectory description [9] which is especially appropriate for analysing experiments with single atoms. Examples are experiments measuring the statistics of macroscopic light and dark periods $[10,11]$ and the spectrum of the light from a three-level atom with a metastable state $[12,13]$. A quantum jump approach was also applied to calculate the spatial interference pattern of the photons spontaneously emitted by two atoms [14] which was observed experimentally by Eichmann et al. [15].

Recently, an experiment was conducted by Eschner et al. $[16,17]$ to measure the fluorescence of a single threelevel barium ion kept at a fixed distance from a mirror. Qualitative explanations were given for most of the effects observed. A recent theoretical study by Dorner and Zoller [18] provides a detailed description of the exper- imental setup considering a two-level atom and a onedimensional model of the free radiation field. Special attention is paid to a regime of large atom-mirror distances where intrinsic memory effects cannot be neglected anymore. In contrast to this we present here an alternative study with a full three-dimensional treatment of the free radiation field where delay time effects are considered negligible. Nevertheless, same qualitative effects resulting from the presence of the mirror as in [18] are predicted and good quantitative agreement with the experimental findings $[16,17]$ is achieved. An earlier experiment by Drexhage [19] in 1974 observed the fluorescence from molecules deposited on mirrors.

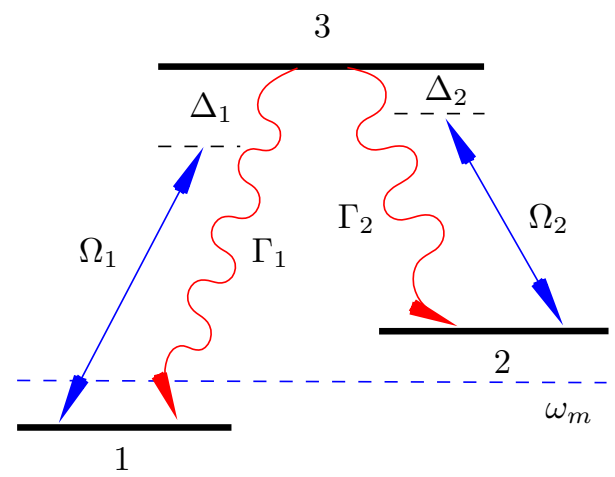

FIG. 1. Atomic level scheme. Two lasers with Rabi frequency $\Omega_{1}$ and $\Omega_{2}$ and detunings $\Delta_{1}$ and $\Delta_{2}$ drive the two transitions in the $\Lambda$ system. The free-space spontaneous decay rates of the upper level are $\Gamma_{1}$ and $\Gamma_{2}$.

In experiment [16], the atom is driven by two detuned laser fields and emits photons along two transitions that comprise a $\Lambda$ system (see Figure 1). In the following, the Rabi frequency and the detuning of the laser field driving the $3-j$ transition $(j=1,2)$ are denoted by $\Omega_{j}$ and $\Delta_{j}$,

*a.beige@mpq.mpg.de 
respectively, while $\Gamma_{1}$ and $\Gamma_{2}$ are the free-space spontaneous decay rates of the upper level. Detectors measure the intensities of the spontaneously emitted photons from the two transitions (see Figure 2). One detector is only sensitive to photons with frequency $\omega_{31}$ and the measured intensity shows a strong sinusoidal dependence on the atom-mirror distance $r$ with maximum visibility of $72 \%$. The other detector, measuring the photons with frequency $\omega_{32}$, which are not affected by the mirror, sees an intensity that depends only weakly on $r$ and has a visibility of about $1 \%$. The maxima of the two light intensities are shifted with respect to each other.

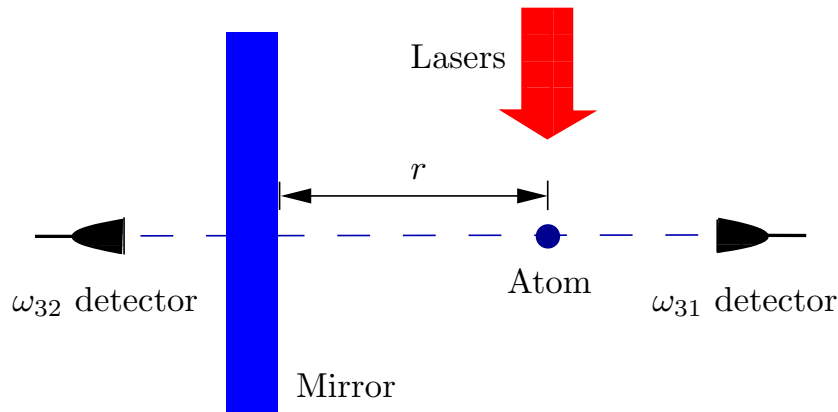

FIG. 2. Experimental setup of an atom placed at a fixed distance $r$ from a mirror and emitting photons with frequencies $\omega_{31}$ and $\omega_{32}$. The mirror is only sensitive to photons with frequency $\omega_{31}$ and two detectors measure light intensities.

Here it is assumed that the lens placed between the atom and the mirror in experiment [16] projects the boundary conditions imposed by the mirror on the free radiation field onto a plane close to the atom. As the atom was located near the focus point of the lens, the experiment can be described by the setup in Figure 2 with an effective atom-mirror distance of the order of the wavelength $\lambda_{31}$. The aim of this paper is to explain the experiment with a quantum mechanical approach. Qualitative and quantitative agreement with the experimental results is obtained.

The paper is organised as follows. Section II presents a quantum jump description of an atom in front of a mirror. The spatial-dependent decay rates and level shifts of the atom are calculated and master equations are derived to find the steady state of the laser-driven atom. These are the ingredients necessary to calculate the intensities of the emitted photons. In Section III we apply our results to the experiment by Eschner et al., while Section IV shows that many aspects of the experiment can also be predicted by means of a mirror-atom model resulting from a comparison of the setup with a classical analog. For example, the effect of the mirror, modifying the overall decay rate of the upper atomic level and introducing an $r$-dependent level shift, can be understood as subradiance between the atom and its mirror image. Finally, an overview of the paper is presented in the conclusions.

\section{QUANTUM MECHANICAL DESCRIPTION}

In this section the atom in front of the mirror is described by the quantum jump approach [9]. The latter consists of two main parts; on the one hand, it gives the time evolution of the atom when no photons are emitted, and on the other, it gives the spatial distribution of the emitted photons depending on the particular state of the atom at the time of the emission. Let us see how this can be obtained from the Schrödinger equation. The corresponding Hamiltonian is of the form

$$
H=H_{\text {atom }}+H_{\text {field }}+H_{\text {laser }}+H_{\text {int }} .
$$

The first three terms are the interaction-free Hamiltonian of the atom, the free radiation field and the classical laser field, while the last term

$$
H_{\mathrm{int}}=e \mathbf{D} \cdot \mathbf{E}(\mathbf{r})
$$

describes the interaction of the atom with the quantised electric field in the dipole approximation. Here $\mathbf{D}$ is the atom dipole operator $\mathbf{D}=\mathbf{D}_{31}|3\rangle\left\langle 1\left|+\mathbf{D}_{32}\right| 3\right\rangle\langle 2|+$ h.c. and $\mathbf{E}(\mathbf{r})$ is the observable of the free radiation field at the position $\mathbf{r}$ of the atom modified by the presence of the mirror. Choosing the coordinate system such that the mirror surface corresponds to the $x=0$ plane, leads to the classical constraint that, at $x=0$, the component of the electric field parallel to the mirror surface has to vanish, i.e.

$$
\mathbf{E}_{\|}(\mathbf{x}: x=0)=0,
$$

for all frequencies that see the mirror. This classical constraint gives at the quantum level a modification on the electric field observable restricting the expectation value of its parallel component to zero on the surface of the mirror [20]. Consider the case where due to the mirror the radiation from the 3-1 transition gets reflected and hence satisfies the constraint (3), while the mirror is transparent for photons from the 3-2 transition. To take this into account a cut-off frequency $\omega_{m}$ is introduced that lies between the typical frequencies $\omega_{31}$ and $\omega_{32}$ of the $\Lambda$ system. The mirror is assumed to be transparent for all frequencies below $\omega_{m}$, and perfectly reflective for frequencies above it. As it is seen later the results derived in this section are independent of the exact value of the chosen cut-off frequency. At an arbitrary position $\mathbf{x}=(x, y, z)$ in the right half space of the mirror (see Figure 2) and with $\mathbf{k}=k_{\|} \hat{\mathbf{k}}_{\|}+k_{x} \hat{\mathbf{x}}$ the electric field observable can then be written as [4]

$$
\begin{aligned}
\mathbf{E}(\mathbf{x})= & \mathrm{i} \sum_{\mathbf{k} \lambda: \omega_{k}<\omega_{m}}\left(\frac{\hbar \omega_{k}}{2 \varepsilon_{0} V}\right)^{1 / 2} \boldsymbol{\epsilon}_{\mathbf{k} \lambda} a_{\mathbf{k} \lambda} \mathrm{e}^{\mathrm{i} \mathbf{k} \cdot \mathbf{x}} \\
& +\mathrm{i} \sum_{\mathbf{k}: \omega_{k} \geq \omega_{m}}\left(\frac{\hbar \omega_{k}}{\varepsilon_{0} V}\right)^{1 / 2}\left[\mathrm{i}\left(\hat{\mathbf{x}} \times \hat{\mathbf{k}}_{\|}\right) \sin k_{x} x a_{\mathbf{k} 1}\right.
\end{aligned}
$$




$$
\begin{aligned}
& \left.+\frac{1}{k}\left(k_{\|} \hat{\mathbf{x}} \cos k_{x} x-\mathrm{i} k_{x} \hat{\mathbf{k}}_{\|} \sin k_{x} x\right) a_{\mathbf{k} 2}\right] \mathrm{e}^{\mathrm{i} \mathbf{k}_{\|} \cdot \mathbf{x}} \\
& + \text { h.c. }
\end{aligned}
$$

where $a_{\mathbf{k} 1}$ and $a_{\mathbf{k} 2}$ are the annihilation operators for photons with polarisation $\epsilon_{\mathbf{k} 1}=\hat{\mathbf{x}} \times \hat{\mathbf{k}}_{\|}$and $\boldsymbol{\epsilon}_{\mathbf{k} 2}=$ $\left(k_{\|} \hat{\mathbf{x}}-k_{x} \hat{\mathbf{k}}_{\|}\right) / k$, respectively, and wave vector $\mathbf{k}$.

From (2) and (4) the effect of the mirror on the atomic fluorescence can be calculated. Assume that the initial state of the atom is known and equals $|\psi\rangle$ while the free radiation field is in the vacuum state $\left|0_{\mathrm{ph}}\right\rangle$. This is an allowed physical state that develops according to the Hamiltonian (1) for a certain time $\Delta t$. If level 3 is populated, this time evolution leads to population of all possible one-photon states [9]. Consider now a detector placed in a certain direction $\hat{\mathbf{k}}$ away from the atom that measures single photons resulting from the $3-j$ transition [14]. To determine the state of the system in case of a click at this detector one has to apply either the projector

$$
\mathbb{P}_{\hat{\mathbf{k}}}^{(1)}=\sum_{k \lambda: \omega_{k} \geq \omega_{m}}\left|1_{k \hat{\mathbf{k}} \lambda}\right\rangle\left\langle 1_{k \hat{\mathbf{k}} \lambda}\right|
$$

if $j=1$, or the projector

$$
\mathbb{P}_{\hat{\mathbf{k}}}^{(2)}=\sum_{k \lambda: \omega_{k}<\omega_{m}}\left|1_{k \hat{\mathbf{k}} \lambda}\right\rangle\left\langle 1_{k \hat{\mathbf{k}} \lambda}\right|
$$

if $j=2$. When a click is registered at a detector, the photon is absorbed and the free radiation field changes to its ground state $\left|0_{\mathrm{ph}}\right\rangle$.

The probability density for a click can be obtained from the norm of the unnormalised state of the system after an emission and equals

$$
I_{\hat{\mathbf{k}}}^{(j)}(\psi)=\lim _{\Delta t \rightarrow 0} \frac{1}{\Delta t} \| \mathbb{P}_{\hat{\mathbf{k}}}^{(j)} U(\Delta t, 0)\left|0_{\mathrm{ph}}\right\rangle|\psi\rangle \|^{2} .
$$

If the coupling constants of the atom to the free radiation field are introduced as

$$
\begin{array}{r}
g_{\mathbf{k} \lambda}^{(1)} \equiv-e\left(\omega_{k} / \varepsilon_{0} \hbar V\right)^{1 / 2} \mathbf{D}_{31} \cdot \boldsymbol{\epsilon}_{\mathbf{k} \lambda} \\
g_{\mathbf{k} \lambda}^{(2)} \equiv \mathrm{i} e\left(\omega_{k} / 2 \varepsilon_{0} \hbar V\right)^{1 / 2} \mathbf{D}_{32} \cdot \boldsymbol{\epsilon}_{\mathbf{k} \lambda}
\end{array}
$$

and the dipole moment $\mathbf{D}_{31}$ is taken for convenience [21] to be parallel to the mirror surface, the interaction Hamiltonian can, with respect to the interactionfree Hamiltonian and within the rotating wave approximation, be written as

$$
\begin{aligned}
H_{\text {int }}^{(\mathrm{I})}= & \hbar \sum_{\mathbf{k} \lambda: \omega_{k}<\omega_{m}} g_{\mathbf{k} \lambda}^{(2)} a_{\mathbf{k} \lambda} \mathrm{e}^{\mathrm{i} \mathbf{k} \cdot \mathbf{r}} \mathrm{e}^{\mathrm{i}\left(\omega_{32}-\omega_{k}\right) t}|3\rangle\langle 2| \\
& +\hbar \sum_{\mathbf{k} \lambda: \omega_{k} \geq \omega_{m}} g_{\mathbf{k} \lambda}^{(1)} a_{\mathbf{k} \lambda} \mathrm{e}^{\mathrm{i} \mathbf{k}_{\|} \cdot \mathbf{r}} \mathrm{e}^{\mathrm{i}\left(\omega_{31}-\omega_{k}\right) t}|3\rangle\langle 1| \sin k_{x} r \\
& + \text { h.c. }
\end{aligned}
$$

Using first-order perturbation theory and the approximations usually applied in quantum optics, (7) leads to

$$
I_{\hat{\mathbf{k}}}^{(1)}(\psi)=\frac{3 \Gamma_{1}}{4 \pi}\left(1-\left|\hat{\mathbf{D}}_{31} \cdot \hat{\mathbf{k}}\right|^{2}\right) P_{3}(\psi) \sin ^{2} k_{31 x} r
$$

for the photons that are affected by the mirror and

$$
I_{\hat{\mathbf{k}}}^{(2)}(\psi)=\frac{3 \Gamma_{2}}{8 \pi}\left(1-\left|\hat{\mathbf{D}}_{32} \cdot \hat{\mathbf{k}}\right|^{2}\right) P_{3}(\psi)
$$

otherwise. Here $\Gamma_{j}$ is the spontaneous emission rate of the atom in free space through the $3-j$ channel, while $P_{3}(\psi)=|\langle 3 \mid \psi\rangle|^{2}$ denotes the initial population in the excited state. This shows that the emission intensity of the 3-1 transition strongly depends on the atom-mirror distance through its proportionality to the factor $\sin ^{2} k_{31 x} r$, while $I_{\hat{\mathbf{k}}}^{(2)}(\psi)$ is not a function of $r$.

It has hitherto been assumed that the atomic state $|\psi\rangle$ is always the same by the time of an emission. This is not the case for the experimental setup in Figure 2, in which the atom is continuously driven by a laser field. To apply our results to this situation, the atom has to be described by the steady-state matrix $\rho^{\mathrm{ss}}$ and $P_{3}(\psi)$ has to be replaced by $P_{3}\left(\rho^{\mathrm{ss}}\right)=\left\langle 3\left|\rho^{\mathrm{ss}}\right| 3\right\rangle$. To calculate the stationary state master equations are employed. They are in general of the form

$$
\dot{\rho}=-\frac{\mathrm{i}}{\hbar}\left[H_{\text {cond }} \rho-\rho H_{\text {cond }}^{\dagger}\right]+\mathcal{R}(\rho) .
$$

Here $H_{\text {cond }}$ is the non-Hermitian Hamiltonian that describes the time evolution of the atom under the condition of no photon emission, while $\mathcal{R}(\rho)$ gives its unnormalised state after an emission. For the atom in front of a mirror, $\mathcal{R}(\rho)$ is given by

$$
\mathcal{R}(\rho)=\sum_{j=1,2} \bar{\Gamma}_{j}|j\rangle\langle 3|\rho| 3\rangle\langle j|
$$

where $\bar{\Gamma}_{1}$ and $\bar{\Gamma}_{2}$ are the modified overall decay rates. They are obtained by integrating $I_{\hat{\mathbf{k}}}^{(j)}(\psi)$ over all directions, which gives by definition $\bar{\Gamma}_{j} P_{3}(\psi)$. This leads for the dipole moment $\mathbf{D}_{31}$ oriented parallel to the mirror, as in [4], to

$$
\bar{\Gamma}_{1}=\Gamma_{1}\left[1-\frac{3}{2}\left(\frac{\sin 2 k_{31} r}{2 k_{31} r}+\frac{\cos 2 k_{31} r}{\left(2 k_{31} r\right)^{2}}-\frac{\sin 2 k_{31} r}{\left(2 k_{31} r\right)^{3}}\right)\right]
$$

and $\bar{\Gamma}_{2}=\Gamma_{2}$. As expected, the decay rate $\bar{\Gamma}_{1}$ is altered by the mirror and (14) is in perfect agreement with the general case presented in [6].

To derive the conditional Hamiltonian $H_{\text {cond }}$ we proceed as above and assume that the effect of the environment and the detectors is the same as the effect of continuous measurements on the free radiation field $[9,14]$. In case no photon is found after the time $\Delta t$, the projector 
onto the field vacuum $\left|0_{\mathrm{ph}}\right\rangle\left\langle 0_{\mathrm{ph}}\right|$ has to be applied to the state of the system. Thus we obtain

$$
\left|0_{\mathrm{ph}}\right\rangle\left\langle 0_{\mathrm{ph}}|U(\Delta t, 0)| 0_{\mathrm{ph}}\right\rangle|\psi\rangle \equiv\left|0_{\mathrm{ph}}\right\rangle U_{\text {cond }}(\Delta t, 0)|\psi\rangle .
$$

Using second-order perturbation theory and the same approximations as above, the no-photon time evolution is summarised within the Hamiltonian $H_{\text {cond }}$, which is, in the Schrödinger picture, given by

$$
H_{\text {cond }}(t)=\sum_{j=1,2} \frac{1}{2} \hbar \Omega_{j} \mathrm{e}^{\mathrm{i}\left(\omega_{3}-\omega_{j}-\Delta_{j}\right) t / \hbar}|j\rangle\langle 3|+\text { h.c. }
$$

$$
\begin{aligned}
P_{3}\left(\rho^{\mathrm{ss}}\right)= & 4\left(\bar{\Delta}_{1}-\bar{\Delta}_{2}\right)^{2}\left(\bar{\Gamma}_{1}+\bar{\Gamma}_{2}\right) \Omega_{1}^{2} \Omega_{2}^{2} \\
\times & \left\{\left[\left(\Omega_{1}^{2}+\Omega_{2}^{2}\right)^{2}+8\left(\bar{\Delta}_{1}-\bar{\Delta}_{2}\right)^{2} \bar{\Gamma}_{1} \bar{\Gamma}_{2}\right]\left(\bar{\Gamma}_{1} \Omega_{2}^{2}+\bar{\Gamma}_{2} \Omega_{1}^{2}\right)+4\left(\bar{\Delta}_{1}-\bar{\Delta}_{2}\right)^{2} \bar{\Gamma}_{1} \bar{\Gamma}_{2}\left(\bar{\Gamma}_{1} \Omega_{1}^{2}+\bar{\Gamma}_{2} \Omega_{2}^{2}\right)\right. \\
& +4\left(\bar{\Delta}_{1}-\bar{\Delta}_{2}\right)^{2}\left[\bar{\Gamma}_{1}^{3} \Omega_{2}^{2}+2\left(\bar{\Gamma}_{1}+\bar{\Gamma}_{2}\right) \Omega_{1}^{2} \Omega_{2}^{2}+\bar{\Gamma}_{2}^{3} \Omega_{1}^{2}\right]-8\left(\bar{\Delta}_{1}-\bar{\Delta}_{2}\right)\left(\bar{\Delta}_{1} \bar{\Gamma}_{1} \Omega_{2}^{4}-\bar{\Delta}_{2} \bar{\Gamma}_{2} \Omega_{1}^{4}\right) \\
& \left.+16\left(\bar{\Delta}_{1}-\bar{\Delta}_{2}\right)^{2}\left(\bar{\Delta}_{1}^{2} \bar{\Gamma}_{1} \Omega_{2}^{2}+\bar{\Delta}_{2}^{2} \bar{\Gamma}_{2} \Omega_{1}^{2}\right)\right\}^{-1},
\end{aligned}
$$

where the notation $\bar{\Delta}_{j} \equiv \Delta_{j}-\Delta$ has been introduced. This result shows that the stationary state of the atom is indeed affected by the presence of the mirror because of its dependence on the decay rate $\bar{\Gamma}_{1}$ and the level shift $\Delta$. Hence, both intensities $I_{\hat{\mathbf{k}}}^{(1)}\left(\rho^{\mathrm{ss}}\right)$ and $I_{\hat{\mathbf{k}}}^{(2)}\left(\rho^{\mathrm{ss}}\right)$ show spatial modulations originating from the boundary condition applied on the electric field observable $\mathbf{E}$.

\section{COMPARISON WITH EXPERIMENTAL RESULTS}

In the experiment by Eschner et al. [16], a lens was employed to enhance the effect of the mirror in the neighborhood of the atom which was placed near the focus point $F$. The lens creates an image of the mirror near the atom, effectively changing the atom-mirror distance. With the same notation as in Figure 3 and classical optics considerations, it is seen that the distance between the mirror image and $F$ is $x=f^{2} / R$. Considering the distances used in the experiment, where $f=12.5 \mathrm{~mm}$ and $R=25 \mathrm{~cm}$, we obtain $x=625 \mu \mathrm{m}$. Since the atom is located close to $F$ it is also located very near the mirror image. Alternatively, one might consider the geometrically equivalent model where the atom is projected by the lens into the neighborhood of the mirror with an effective distance $r$ from it which can be made to be of the order of the wavelength $\lambda_{31}=493 \mathrm{~nm}$ [22]. where

$$
\Delta=\frac{3 \Gamma_{1}}{4}\left(\frac{\cos 2 k_{31} r}{2 k_{31} r}-\frac{\sin 2 k_{31} r}{\left(2 k_{31} r\right)^{2}}-\frac{\cos 2 k_{31} r}{\left(2 k_{31} r\right)^{3}}\right)
$$
atomic state $|3\rangle$ resulting from the modification of the free radiation field due to the presence of the mirror.

From (12), (13) and (16) and the condition $\dot{\rho}^{\mathrm{ss}}=0$ the expression for the steady-state population of the excited state, $P_{3}\left(\rho^{\mathrm{ss}}\right)$, is obtained: is, in agreement with [6], the level shift of the excited

$$
+\hbar \Delta|3\rangle\left\langle 3\left|-\frac{\mathrm{i}}{2} \hbar\left(\bar{\Gamma}_{1}+\bar{\Gamma}_{2}\right)\right| 3\right\rangle\langle 3|,
$$


was oriented parallel to the mirror surface.

Figure 4 shows the intensities $I_{\hat{\mathbf{k}}}^{(1)}\left(\rho^{\mathrm{ss}}\right)$ and $I_{\hat{\mathbf{k}}}^{(2)}\left(\rho^{\mathrm{ss}}\right)$ as a function of $r$ where the relevant parameters have been taken from [16]. As expected, the photons which see the mirror show a very strong sinusoidal $r$-dependence. If the effective atom-mirror distance $r$ is of the order of the wavelength $\lambda_{31}$, then the intensity measured by a detector behind the mirror also shows an $r$-dependence. Nevertheless, this dependence is much weaker and vanishes for large $r$. The relative order of magnitude of the intensities presented in Figure 4, assuming $r \sim 5 \lambda_{31}$, is in agreement with the experimental findings (see Figure 3 in [16]).

(a)

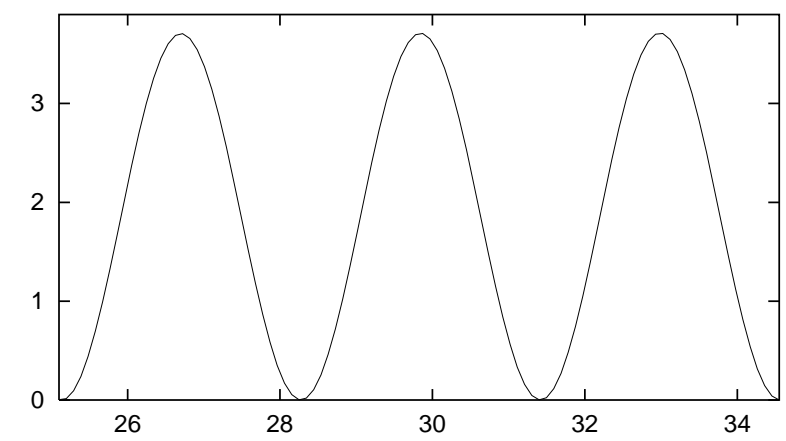

(b)

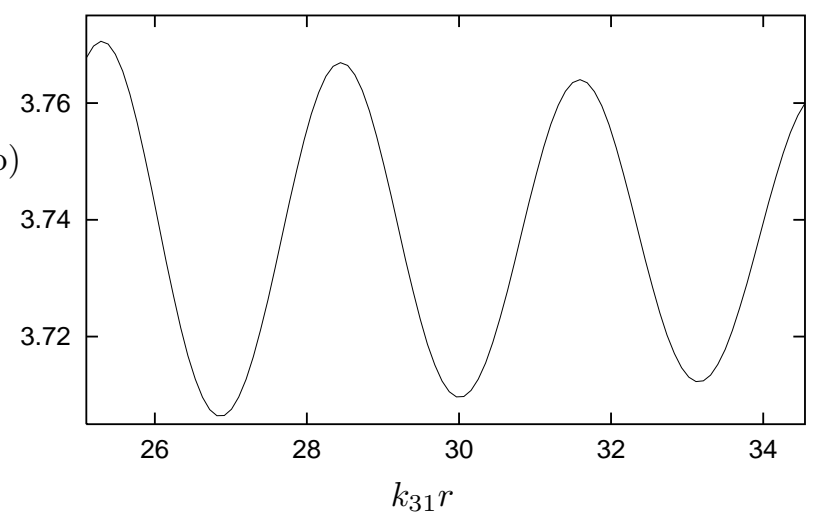

FIG. 4. The intensities, $I_{\hat{\mathrm{k}}}^{(1)}\left(\rho^{\mathrm{ss}}\right)$ (a) and $I_{\hat{\mathrm{k}}}^{(2)}\left(\rho^{\mathrm{ss}}\right)$ (b) as a function of the effective atom-mirror distance for $\Delta_{1}=2 \mathrm{MHz}, \Delta_{2}=0, \Omega_{1}=10 \mathrm{MHz}, \Omega_{2}=5 \mathrm{MHz}$, $\Gamma_{1}=15.1 \mathrm{MHz}$ and $\Gamma_{2}=5.4 \mathrm{MHz}$. The vertical axis is given in units of $10^{-2} \mathrm{MHz}$ for both plots.

In addition, Figure 4 shows that the two intensities can be anticorrelated, having a phase difference close to $\pi$. This effect is due to their difference in nature and can vary for different values of the $\Omega_{i}$ and $\Delta_{i}$ parameters. The origin of the pattern in Figure 4(a) is the $\sin ^{2} k_{x} r$ factor in $I_{\hat{\mathbf{k}}}^{(1)}\left(\rho^{\mathrm{ss}}\right)$, while the $r$-dependence of the population $P_{3}\left(\rho^{\mathrm{ss}}\right)$ is in this case insignificant. In contrast, the pattern shown in Figure $4(\mathrm{~b})$ is only due to the $P_{3}\left(\rho^{\mathrm{ss}}\right)$ dependence of $I_{\hat{\mathbf{k}}}^{(2)}\left(\rho^{\mathrm{ss}}\right)$. Its spatial configuration is dictated by $\bar{\Gamma}_{1}$, which includes the dominant term $\sin 2 k_{31} r$, and by $\Delta$, which includes the dominant term $\cos 2 k_{31} r$.
Hence, the first plot is a consequence of the modification of the electromagnetic field observable in the neighborhood of the atom, while the second plot is a consequence of the modification of the spontaneous emission rate $\bar{\Gamma}_{1}$ and the level shift $\Delta$ of the excited atomic level. From this we can deduce that the anticorrelation of the intensities takes place only for certain values of the Rabi frequencies and detunings.

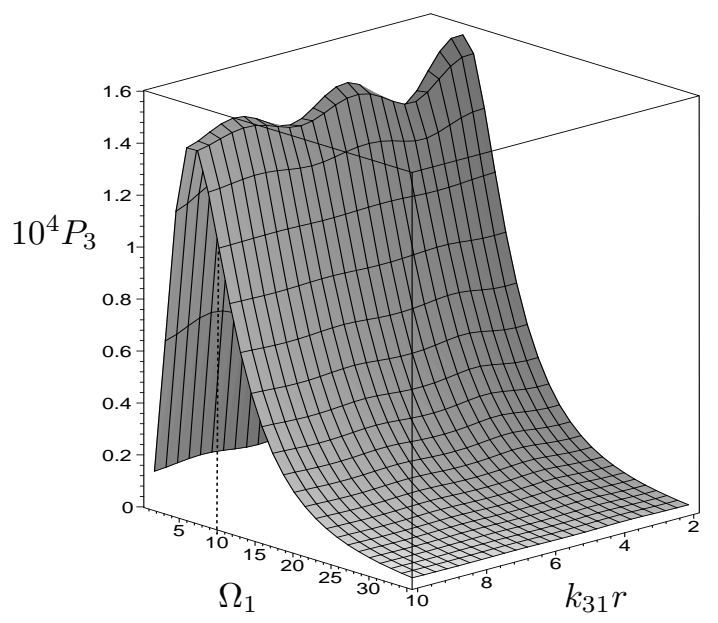

FIG. 5. Steady state population of the excited state, $P_{3}\left(\rho^{\mathrm{ss}}\right)$, as a function of the Rabi frequency $\Omega_{1}$ (in $\mathrm{MHz}$ ) and the atom-mirror distance $r$ for $\Delta_{1}=0, \Delta_{2}=0.1 \mathrm{MHz}$ and $\Omega_{2}=10 \mathrm{MHz}$. For $\Omega_{1}=\Omega_{2}$ the spatial modulations disappear (see dotted line). The $\pi$ phase change is apparent above and below this value.

It is instructive to have a closer look at the modulation of $P_{3}\left(\rho^{\mathrm{ss}}\right)$ as a function of the Rabi frequencies and laser detunings. For example, for detunings $\Delta_{1}$ and $\Delta_{2}$ much smaller than the Rabi frequencies of the driving laser fields relation (18) simplifies to

$$
P_{3}\left(\rho^{\mathrm{ss}}\right) \approx 4\left(\Delta_{1}-\Delta_{2}\right)^{2} \frac{\Omega_{1}^{2} \Omega_{2}^{2}}{\left(\Omega_{1}^{2}+\Omega_{2}^{2}\right)^{2}} \frac{\bar{\Gamma}_{1}+\bar{\Gamma}_{2}}{\bar{\Gamma}_{1} \Omega_{2}^{2}+\bar{\Gamma}_{2} \Omega_{1}^{2}} .
$$

The second factor gives the main modulation of the intensity with respect to the Rabi frequencies, while the third factor gives the distance-dependent oscillations observed in Figure 4(b). The latter modulation may change phase by $\pi$ if the ratio $\Omega_{1} / \Omega_{2}$ changes from smaller than one to larger than one, as can be predicted by (19) and Figure 5. In particular, if $\Omega_{1}=\Omega_{2}$ then the modulations with $r$ vanish. On the other hand, the maximum amplitude of the fringes appears for laser intensities for which also $I_{\hat{\mathbf{k}}}^{(2)}\left(\rho^{\mathrm{ss}}\right)$ becomes maximal. For $\Delta_{1}=\Delta_{2}$, the population $P_{3}\left(\rho^{\mathrm{ss}}\right)$ vanishes as a dark state is generated between the levels 1 and 2. Trapping of the population to a single ground state also occurs when one of the Rabi frequencies becomes much larger than the other. 
Taking into account that experimentally a maximum visibility of $72 \%$ has been found for $I_{\hat{\mathrm{k}}}^{(1)}\left(\rho^{\mathrm{ss}}\right)$, one can predict the reduction of the visibility $V$ for increasing Rabi frequencies from Figure 5. In Eschner et al. it was argued that the reduction of the maximum visibility from unity is mainly due to thermal motion of the ion, non-optimal cooling conditions, fluctuations of the atom-mirror distance and imperfect mapping of the mirror neighborhood to the neighborhood of the atom by the lens. In addition, it was observed that the visibility was greater than $50 \%$ for Rabi frequencies $\Omega_{1}$ below saturation, while it reduced to below $10 \%$ when the Rabi frequency increased to 3 -fold saturation. Indeed, from a figure similar to Figure 5, but for $\Omega_{2} \sim 1 \mathrm{MHz}$, we see that at 3 -fold saturation the population of level 3 and hence the amplitude of the oscillations of the intensity $I_{\hat{\mathrm{k}}}^{(1)}\left(\rho^{\mathrm{ss}}\right)$ reduce by about 30 times from their value at the saturation point. This can explain the $\Omega_{1}$-dependent reduction of the visibility observed experimentally.

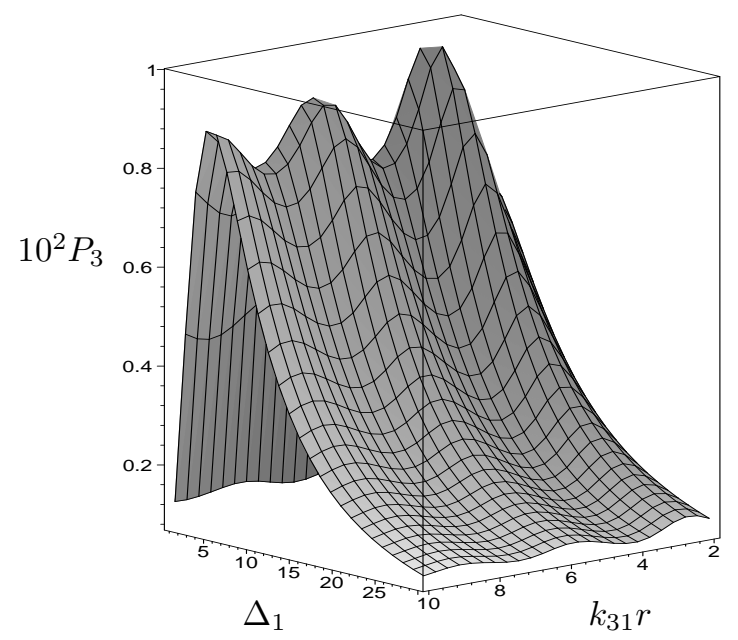

FIG. 6. Steady state population of the excited state, $P_{3}\left(\rho^{\mathrm{ss}}\right)$, as a function of the detuning $\Delta_{1}$ (in $\mathrm{MHz}$ ) and the atom-mirror distance $r$ for $\Delta_{2}=0, \Omega_{1}=1 \mathrm{MHz}$ and $\Omega_{2}=10 \mathrm{MHz}$.

In Figures 6 we see the continuous phase change of the maxima of the population $P_{3}\left(\rho^{\mathrm{ss}}\right)$ when the detuning $\Delta_{1}$ is varying. In particular, if we take the detunings much larger than the Rabi frequencies and decay rates, we obtain

$$
P_{3}\left(\rho^{\mathrm{ss}}\right) \approx \frac{1}{4} \Omega_{1}^{2} \Omega_{2}^{2} \frac{\bar{\Gamma}_{1}+\bar{\Gamma}_{2}}{\bar{\Delta}_{1}^{2} \bar{\Gamma}_{1} \Omega_{2}^{2}+\bar{\Delta}_{2}^{2} \bar{\Gamma}_{2} \Omega_{1}^{2}},
$$

that gives an indication of the main terms which contribute to the continuous phase change of the $r$ dependent oscillations when one of the detunings is varying. Summarising this, by scanning different Rabi frequencies and detunings it should be possible to observe the variations in the amplitude of the population of level 3 as well as the discrete $\pi$ change or continuous change in the phase of the spatial modulations. In this way one could further verify our description of experimental setup [16].

\section{THE MIRROR-ATOM MODEL}

Describing the setup in Figure 2 in a classical manner, one assumes that the atom is a point-like source with dipole characteristics. As it is classically possible to replace the mirror by a mirror-source at the distance $2 r$, it could be assumed that the radiation properties of the atom can be predicted by replacing the mirror in the quantum setup by a mirror-atom. Indeed, both descriptions lead to the same dependence of the light intensity on the source-mirror distance as found for $I_{\hat{\mathbf{k}}}^{(1)}(\psi)$ in (10). The mirror-atom model [4] can even be used to predict further aspects of experiment [16]. If the atom is initially prepared in the excited state $|3\rangle$ one has $P_{3}(\psi)=1$ and (14) gives the probability density for a photon emission. The quantum theory of dipole-interacting atoms is well known and a comparison with [2] reveals that (14) coincides exactly with the decay rate of two dipole-interacting atoms prepared in the antisymmetric Dicke state of two two-level atoms at a distance $2 r$. In addition, the level shift of $|3\rangle$ given in (17) equals the level shift of the antisymmetric state resulting from the dipole-dipole interaction.

Nevertheless, the mirror-atom model can no longer be used when the state of the atom by the time of the emission has no simple classical analog. It is not possible to take into account the driving of the two atomic transitions by a laser field. Dipole-interacting atoms have a richer structure of internal states and hence they cannot give completely equivalent results with the atom-mirror system.

\section{CONCLUSIONS}

This paper presents a full quantum mechanical study of the fluorescence of an atom in front of a mirror, based on the assumption that the mirror imposes boundary conditions on the electric field observable. In this way, the presence of the mirror affects the interaction of the atom with the free radiation field. This leads to a sinusoidal dependence of the intensities of the emitted light on the atom-mirror distance $r$. In addition, the overall decay rate of the atom becomes a function of $r$ and an $r$-dependent level shift is induced if $r$ is of comparable size to the wavelength of the emitted photons - an effect which can be interpreted in terms of subradiance due to dipole-dipole interaction between the atom and its mirror image. 
In the actual experiment by Eschner et al. [16], the $25 \mathrm{~cm}$ distance between the atom and the mirror was much larger than the wavelength of the emitted photons and a lens was placed near the atom to enhance the effect of the mirror. Motivated by this, a recent paper by Dorner and Zoller [18] took into account time-of-flight effects using a one-dimensional description of the free radiation field. Atom-mirror distances much larger than an optical wavelength were considered and delay differential equations were derived. Similar effects resulting from the presence of the mirror have been predicted, i.e. a sinusoidal dependence of the spontaneous decay rate and the level shift of the upper atomic level on the atom-mirror distance. In contrast to the results presented here those modifications are persisting for large distances due to the one-dimensional character of their model making it difficult to derive quantitative predictions comparable to the experimental findings.

In this paper it was assumed that the lens projects the mirror surface close to the atom so that the atom-mirror distance effectively becomes of similar size as the relevant wavelength. For the simplified setup, including only the atom and the mirror, a full three-dimensional description was given. Good qualitative and quantitative agreement was found with respect to different aspects of the experiment. Delay-time effects were neglected assuming that the relevant time scale for the projection of the mirror to the other side of the lens is in the experiment with about $1.7 \mathrm{~ns}$ [16] sufficiently smaller than the time scale on which the detector performs measurements on the free radiation field. This time scale has been denoted $\Delta t$ in Section II and is restricted from above only by the inverse decay rate of the relevant atomic transition [9] which equals $1 / \Gamma_{1}=416 \mathrm{~ns}$.

Acknowledgments. We would like to thank U. Dorner and B.-G. Englert for interesting and helpful discussions. This work was supported by the European Union through IST-1999-13021-Project QUBITS.

[1] W. Neuhauser, M. Hohenstatt, P. E. Toschek, and H. Dehmelt, Phys. Rev. A 22, 1137 (1980); F. Diedrich and H. Walther, Phys. Rev. Lett. 58, 203 (1987).

[2] R. Dicke, Phys. Rev. 93, 99 (1954); R. H. Lehmberg, Phys. Rev. A 2, 883 (1970); 2, 889 (1970); G. S. Agarwal, Quantum Optics, Springer Tracts of Modern Physics 70 (Springer-Verlag, Berlin 1974).

[3] E. Fermi, Rev. Mod. Phys. 4, 87 (1932); H. B. G. Casimir and D. Bolder, Phys. Rev. 73, 360 (1948); G. Barton, J. Phys. B 7, 2134 (1974).

[4] P. W. Milonni and P. L. Knight, Opt. Commun. 9, 119 (1973); P. W. Milonni, The quantum vacuum: an in- troduction to quantum electrodynamics, Academic Press, Inc., San Diego (1994), Chapter 6.2.

[5] For further studies see for example J. M. Wylie and G. E. Sipe, Phys. Rev. A 30, 1185 (1984); J. M. Wylie and G. E. Sipe, Phys. Rev. A 32, 2030 (1995); B. S.-T. Wu and C. Eberlein, Proc. Roy. Soc. A 455, 2487 (1999); R. Matloob, Phys. Rev. A 62, 022113 (2000) and references therein.

[6] D. Meschede, W. Jhe, and E. A. Hinds, Phys. Rev. A 41, 1587 (1990); E. A. Hinds and V. Sandoghdar, Phys. Rev. A 43, 398 (1991).

[7] H. Walther, Phys. Rep. 219, 263 (1992); Cavity Quantum Electrodynamics, Special Issue of Advances in Atomic, Molecular and Optical Physics, ed. by P. R. Berman, Academic Press Inc. (San Diego, 1994); E. A. Hinds, Adv. Mol. Opt. Phys. 2, 1 (1994).

[8] For a recent cavity experiment see for example G. R. Guthörlein, M. Keller, K. Hayasaka, W. Lange, and H. Walther, Nature 414, 49 (2002).

[9] H. Carmichael, An Open System Approach to Quantum Optics, Lecture Notes in Physics, Springer, Berlin 1992; J. Dalibard, Y. Castin, and K. Mølmer, Phys. Lett. 68, 580 (1992); G. C. Hegerfeldt, Phys. Rev. A 47, 449 (1993). For a recent review see M. B. Plenio and P. L. Knight, Rev. Mod. Phys. 70, 101 (1998).

[10] W. Nagourney, J. Sandberg, and H. Dehmelt, Phys. Rev. Lett. 56, 2797 (1986); Th. Sauter, W. Neuhauser, R. Blatt, and P. E. Toschek, Phys. Rev. Lett. 57, 1696 (1986); J. C. Bergquist, R. G. Hulet, W. M. Itano, and D. J. Wineland, Phys. Rev. Lett. 57, 1699 (1986).

[11] A. Beige and G. C. Hegerfeldt, J. Phys. A 30, 1323 (1997); A. Beige and G. C. Hegerfeldt, Phys. Rev. A 59, 2385 (1999).

[12] V. Bühner and C. Tamm, Phys. Rev. A 61, 061801 (2000).

[13] G. C. Hegerfeldt and M. B. Plenio, Phys. Rev. A 53, 1164 (1996).

[14] C. Schön and A. Beige, Phys. Rev. A 64, 023806 (2001); A. Beige, C. Schön, and J. Pachos, Fortschr. Phys. 50, 594 (2002).

[15] U. Eichmann, J. C. Bergquist, J. J. Bollinger, J. M. Gilligan, W. M. Itano, D. J. Wineland, and M. G. Raizen, Phys. Rev. Lett. 70, 2359 (1993).

[16] J. Eschner, Ch. Raab, F. Schmidt-Kaler, and R. Blatt, Nature 413, 495 (2001).

[17] J. Eschner, Ch. Raab, P. Bouchev, F. Schmidt-Kaler, and R. Blatt, Single ions interfering with their mirror images, World Scientific (in press).

[18] U. Dorner and P. Zoller, Laser-driven atoms in halfcavities, quant-ph/0203147. See also references therein.

[19] K. H. Drexhage, Sci. Am. 222, 108 (1970); K. H. Drexhage, Prog. Opt. 12 (1974).

[20] K. Sundermeyer, Constrained Dynamics, Lecture Notes in Physics 169, Springer Verlag (1982).

[21] This choice of the orientation of the dipole moment maximises the contrast of the observed fluorescence as a function of $r$. The general case of the arbitrary dipole orientation can be treated analogously $[4,6,5]$, but we assume here that the laser driving the $3-1$ transition is chosen such that it excites only an electric dipole moment oriented parallel to the mirror. 
[22] Note that a small displacement of the atom corresponds to an amplified displacement of the image of the atom near the mirror by a few orders of magnitude. Because of this scaling the experiment requires very efficient cooling of the atomic vibration. 